\title{
Rectal Ulcer in a Patient with VZV Sacral Meningoradiculitis (Elsberg Syndrome)
}

\author{
Hideyuki Matsumoto ${ }^{1}$, Takahiro Shimizu ${ }^{1}$, Shin-ichi Tokushige ${ }^{1}$, Hideo Mizuno ${ }^{2}$, \\ Yukifusa Igeta ${ }^{1}$ and Hideji Hashida ${ }^{1}$
}

\begin{abstract}
This report describes the case of a 55-year-old woman with varicella-zoster virus (VZV) sacral meningoradiculitis (Elsberg syndrome) who presented with herpes zoster in the left S2 dermatome area, urinary retention, and constipation. Lumbar magnetic resonance imaging showed the left sacral nerve root swelling with enhancement. Thereafter, she suddenly showed massive hematochezia and hemorrhagic shock because of a rectal ulcer. To elucidate the relation between Elsberg syndrome and rectal ulcer, accumulation of similar cases is necessary. To avoid severe complications, attention must be devoted to the possibility of rectal bleeding in the early stage of Elsberg syndrome.
\end{abstract}

Key words: Elsberg syndrome, urinary retention, varicella-zoster virus, rectal ulcer

(Intern Med 51: 651-654, 2012)

(DOI: 10.2169/internalmedicine.51.6514)

\section{Introduction}

In 1931, Elsberg described a syndrome characterized by rapidly developing sacral root deficits with sphincter dysfunction. In cerebrospinal fluid (CSF), pleocytosis and elevated protein are often observed. Most probably, the basis of this condition is localized radiculitis of the sacral roots (1). Now, lumbosacral meningoradiculitis associated with urinary retention is well known as Elsberg syndrome (1-7). Meningitis, radiculitis, or both are regarded as causing sacral motor and sensory neuropathy with dysautonomia, inducing sphincter dysfunction (1). This syndrome is often induced by sacral herpes such as herpes simplex virus (HSV) and varicella-zoster virus (VZV). Patients with this syndrome frequently show several symptoms such as urinary retention, constipation, impotence, and sensory disturbance in legs, although most of them mainly suffer from urinary retention and usually need transient urethral catheter (1-7).

In papers related to this syndrome, however, the possibility of lower gastrointestinal bleeding has been little recognized. This report describes the case of a patient with VZV sacral meningoradiculitis (Elsberg syndrome) who presented with hemorrhagic shock because of rectal ulcer.

\section{Case Report}

The patient was a 55-year-old woman who had a painful rash on the hip and leg of the left side. Two days later, she complained of dysuria and constipation. On the following day, she was admitted to our hospital because she was unable to urinate or defecate. She had no symptoms of meningitis such as headache, nausea, or vomiting.

She had no remarkable prior history, and no history of constipation. Her family history was also unremarkable. She had smoked 20 cigarettes per day for 35 years, but she had only rarely drunk alcohol. She had taken no medications.

On admission, her blood pressure was 140/55 Torr, her pulse was 72 beats/min, and her body temperature was $36.8^{\circ} \mathrm{C}$. Physical and neurological examinations revealed erythematous vesicular rashes in the left second sacral spinal (S2) dermatome with spontaneous pain and hyperalgegia, urinary retention, dyschezia, and decreased Achilles tendon reflex on the left side. Desire to urinate and defecate were diminished. Bowel sounds were absent. Meningeal irritation sign was negative. Routine blood examination showed leu-

${ }^{1}$ Department of Neurology, Japanese Red Cross Medical Center, Japan and ${ }^{2}$ Department of Gastroenterology, Japanese Red Cross Medical Center, Japan

Received for publication September 6, 2011; Accepted for publication November 30, 2011

Correspondence to Dr. Hideyuki Matsumoto, hideyukimatsumoto.jp@gmail.com 

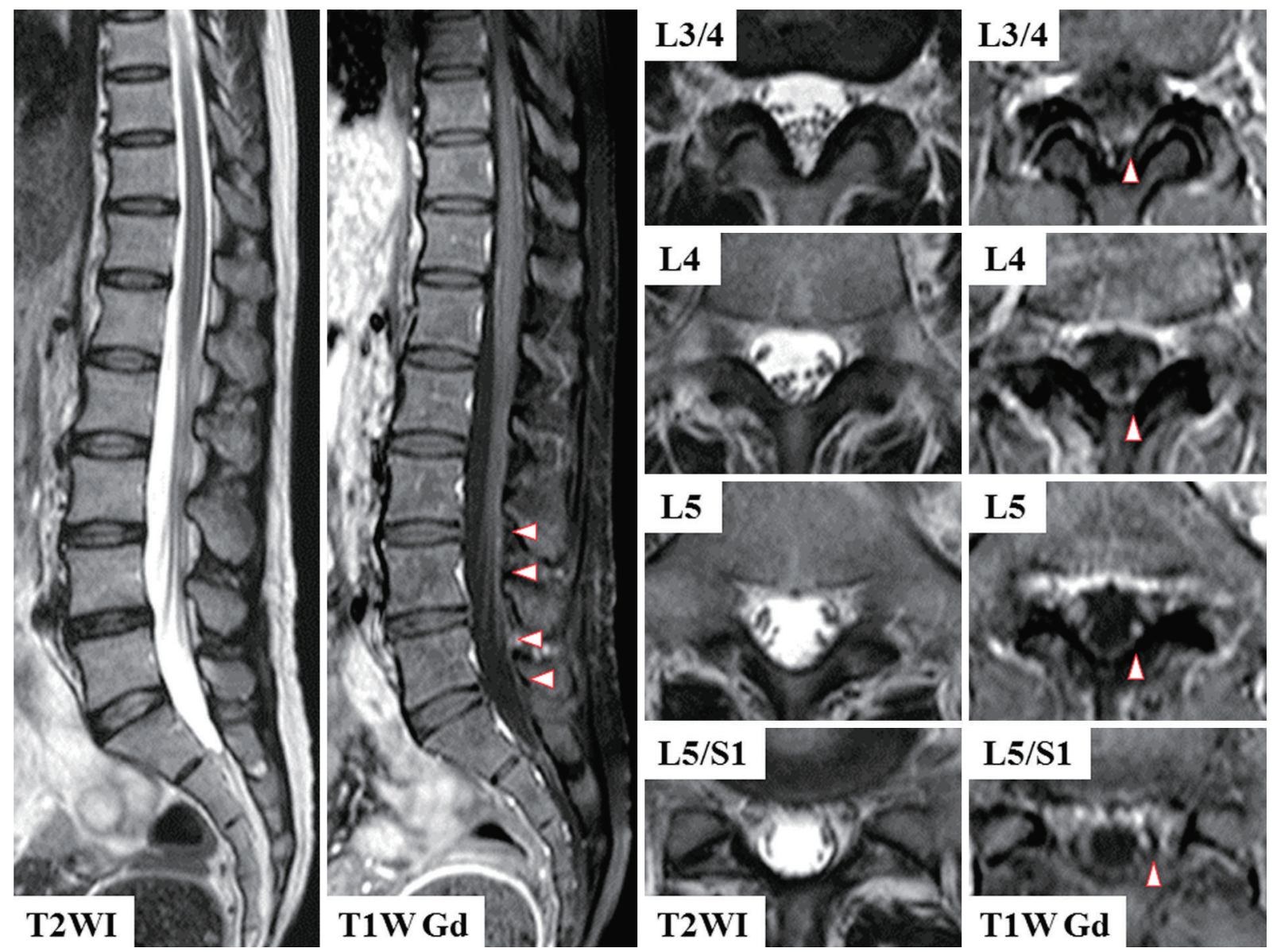

Figure 1. Lumbar MRI in VZV sacral meningoradiculitis. Sagittal T2-weighted image (T2WI) and post-gadolinium (Gd) T1-weighted image (T1WI) are displayed in the left panel, and axial T2WI and post-Gd T1WI in the right panel. MRI clearly portrays left sacral nerve root swelling with Gd enhancement (arrowhead).

kocytosis, positive C-reactive protein and normal albumin (WBC $13,700 / \mu \mathrm{L}$, CRP $0.38 \mathrm{mg} / \mathrm{dL}$, albumin $4.3 \mathrm{~g} / \mathrm{dL}$ ). A blood examination for infectious disease revealed an abnormally high titer of immunoglobulin (Ig) G of VZV (VZV$\operatorname{IgG}>128$ ). Liquor examination showed pleocytosis (cell $130 / \mathrm{mm}^{3}$, poly. $1 \%$, mono. $99 \%$ ), increased protein (protein $53 \mathrm{mg} / \mathrm{dL}$ ), normal glucose (liquor glucose $54 \mathrm{mg} / \mathrm{dL}$, blood glucose $90 \mathrm{mg} / \mathrm{dL}$ ), positive VZV-IgG, and negative HSVIgG [VZV-IgG 7.32 (>2.0), HSV-IgG $0.97(<2.0)$ ]. Lumbar magnetic resonance image (MRI) with gadolinium enhancement revealed left sacral nerve root swelling (Fig. 1). Based on the characteristic skin rash, we made a diagnosis of herpes zoster. Moreover, based on the urinary retention symptom, pleocytosis and elevated protein in CSF, the positive VZV-IgG in both blood and liquor examinations, and the left sacral nerve root involvement on MRI, we made a diagnosis of VZV sacral meningoradiculitis: Elsberg syndrome. Intravenous acyclovir was started for the VZV infection with a dose of $500 \mathrm{mg}$ three times daily. In addition, a urethral catheter was indwelled for urinary retention and oral purgatives were prescribed for dyschezia. Nevertheless, she remained unable to defecate.

At three days after admission, massive hematochezia oc- curred along with defecation of a hard fecal mass; she showed hemorrhagic shock. Her hemoglobin dropped from baseline of $15.8 \mathrm{mg} / \mathrm{dL}$ to $11.4 \mathrm{mg} / \mathrm{dL}$ within one day. Large-volume fluid replacement therapy and red blood cell transfusion were conducted for hemorrhage. Colonoscopy revealed a large round ulcer in the rectum (Fig. 2A). Other portions were intact. Immediately, rectal re-bleeding was prevented by colonoscopic clips (Fig. 2B).

Thereafter, herpes zoster, urinary retention, and dyschezia were gradually diminished. At two weeks after admission, she was discharged with no symptoms. Throughout the admission, no suppository was used.

\section{Discussion}

This report described the case of a 55-year-old woman with VZV sacral meningoradiculitis (Elsberg syndrome) who underwent hemorrhagic shock because of rectal ulcer. Lower gastrointestinal bleeding in association with Elsberg syndrome has not been reported in the relevant literature. Many possibilities related to the cause of rectal ulcer exist, although the cause cannot be identified. Stercoral ulcer, acute hemorrhagic rectal ulcer, VZV related rectal ulcer, and other 


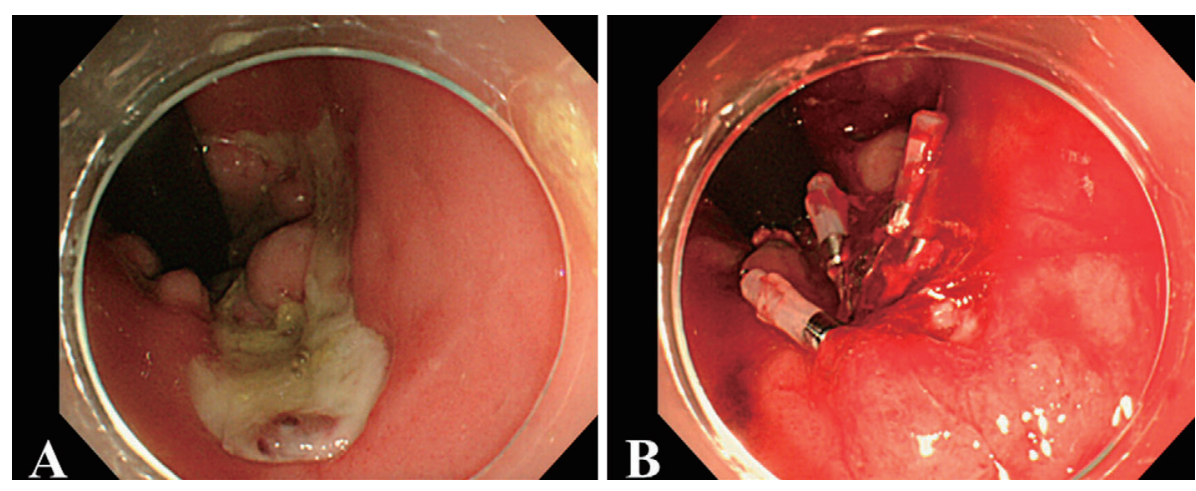

Figure 2. Colonoscopic findings of the rectal ulcer. Colonoscopy revealed the rectal ulcer with an exposed blood vessel (A). The exposed blood vessel in the ulcer was clipped endoscopically (B).

possible causes should be considered.

Stercoral ulcer is one possible cause. The rectal ulcer might result from the acute severe dyschezia caused by Elsberg syndrome. Generally, stercoral ulcer results from impaction of a hard fecal mass on the colonic wall and it induces lower gastrointestinal bleeding $(8,9)$. The most common site of involvement is the rectum, and the lesion is isolated. Constipation invariably precedes the occurrence of the ulcer, and the initial symptom is generally massive hematochezia of sudden onset. Massive blood transfusion is often required (10). These characteristics are compatible with those in the present case. Similar to the mechanism of urinary retention, the anal sphincter dysfunction might be related. However, stercoral ulcer usually occurs in patients older than 70 years of age and it is associated with diseases such as heart failure under hemodialysis, cerebral hemorrhage, and postoperative states, requiring a long period of bed rest (10). However, the present case had no risk factors of stercoral ulcer except for Elsberg syndrome. Indeed, she had performed normal activities of daily living without malnutrition. Moreover, it is questionable whether constipation lasting only five days can produce such a rectal ulcer. Therefore, this possibility remains uncertain.

As another possibility, the pathogenesis in the present case resembles that in acute hemorrhagic rectal ulcer because acute hemorrhagic rectal ulcer occurs suddenly with painless, massive, fresh rectal bleeding, although it occurs consistently in elderly people and in bed-ridden patients who have a severe primary disease (11). Several hypotheses have been presented for the pathogenesis of acute hemorrhagic rectal ulcer, such as stress, thrombus, sepsis, arteriosclerosis, and disturbance of microcirculation. Although the woman described in this report was not bedridden, we cannot exclude the possibility that the pain and mental stress associated with herpes zoster might cause a similar pathogenesis to that found in acute hemorrhagic rectal ulcer.

As an interesting possibility, we propose VZV-related rectal ulcer. Direct VZV invasion into intestinal mucosa or the indirect VZV effect on intestinal mucosa might induce rectal ulcer. VZV can become latent in the nerve cell bodies and less frequently in non-neuronal satellite cells of the dorsal root, cranial nerve, or autonomic ganglion (12). Similar to skin lesions, upon reactivation, VZV might break out of nerve cell bodies and travel down nerve axons to cause viral infection of the skin and intestinal mucosa in the region of the nerve, which might generate gastrointestinal bleeding directly or indirectly.

Other symptoms might be present, such as bowel dysautonomia caused by Elsberg syndrome. Whatever the mechanism, to elucidate the relation between Elsberg syndrome and rectal ulcer, the accumulation of information from similar cases is necessary.

In the present case, the rectal re-bleeding was prevented by the colonoscopic clips. However, if endoscopic therapy is ineffective for the rectal ulcer presenting with massive hematochezia, emergent surgical resection might be necessary (9). To avoid severe complications, we should devote attention to the possibility of rectal bleeding during the early stage of Elsberg syndrome. Our case presented with herpes zoster in the left S2 dermatome area. It was apparently related to the MRI findings showing left sacral nerve root swelling with enhancement. The MRI findings in Elsberg syndrome due to VZV have been less well known, although we consider that the MRI findings are helpful for diagnosis.

In conclusion, Elsberg syndrome can cause rectal ulcer with massive hemorrhage even in a patient with no other disease. To elucidate the relation between Elsberg syndrome and rectal ulcer, information from similar cases must be accumulated. To avoid severe complications, attention should be given to the possibility of rectal bleeding at the early stage in Elsberg syndrome.

\section{The authors state that they have no Conflict of Interest (COI).}

\section{References}

1. Sakakibara R, Yamanishi T, Uchiyama T, Hattori T. Acute urinary retention due to benign inflammatory nervous diseases. J Neurol 253: 1103-1110, 2006.

2. Hemrika DJ, Schutte MF, Bleker OP. Elsberg syndrome: a neurologic basis for acute urinary retention in patients with genital herpes. Obstet Gynecol 68: 37S-39S, 1986. 
3. Eberhardt O, Küker W, Dichgans J, Weller M. HSV-2 sacral radiculitis (Elsberg syndrome). Neurology 63: 758-794, 2004.

4. Yoritaka A, Ohta K, Kishida S. Herpetic lumbosacral radiculoneuropathy in patients with human immunodeficiency virus infection. Eur Neurol 53: 179-181, 2005.

5. Sakakibara R, Uchiyama T, Liu Z, et al. Meningitis-retention syndrome. An unrecognized clinical condition. J Neurol 252: 14951499, 2005

6. Furugen M, Yamashiro S, Tamayose M, et al. Elsberg syndrome with eosinophilic meningoencephalitis caused by Angiostrongylus cantonensis. Intern Med 45: 1333-1336, 2006.

7. Suarez-Calvet M, Rojas-Garcia R, Querol L, Sarmiento LM, Domingo P. Polyradiculoneuropathy associated to human herpesvirus 2 in an HIV-1-infected patient (Elsberg syndrome): case report and literature review. Sex Transm Dis 37: 123-125, 2010.
8. Grinvalsky HT, Bowerman CI. Stercoraceous ulcers of the colon: relatively neglected medical and surgical problem. J Am Med Assoc 171: 1941-1946, 1959.

9. Madan P, Bhayana S, Chandra P, Hughes JI. Lower gastrointestinal bleeding: association with sevelamer use. World J Gastroenterol 14: 2615-2616, 2008.

10. Tada M, Shimizu S, Kawai K. Clinical investigation of stercoral ulcer. Jpn J Geriatr 27: 718-723, 1990 (in Japanese, Abstract in English).

11. Takeuchi K, Tsuzuki $Y$, Ando $T$, et al. Clinical characteristics of acute hemorrhagic rectal ulcer. J Clin Gastroenterol 33: 226-228, 2001.

12. Johnson RW, Dworkin RH. Treatment of herpes zoster and postherpetic neuralgia. BMJ 326: 748-750, 2003.

(C) 2012 The Japanese Society of Internal Medicine http://www.naika.or.jp/imindex.html 